\title{
Situs inversus totalis with a mature teratoma of the posterior mediastinum: A case report
}

\author{
YU QI, CHUNYANG ZHANG, DONGLEI LIU, YANG YANG and SONG ZHAO
}

Department of Thoracic Surgery, The First Affiliated Hospital of Zhengzhou University, Zhengzhou, Henan 450052, P.R. China

Received October 31, 2014; Accepted October 21, 2015

DOI: $10.3892 / 01.2015 .3994$

\begin{abstract}
Situs inversus totalis is an unusual disease with a low incidence. The co-existence of situs inversus totalis with a teratoma is extremely rare. To the best of our knowledge, there is no data on the association between situs inversus totalis and teratoma in the literature. The present study therefore describes the first such case in a 23 -year-old female. The patient presented with intermittent chest tightness, which had lasted for $\sim 3$ years. Following radiological examination, the patient was diagnosed with situs inversus totalis and a mature teratoma in the posterior mediastinum. Surgical resection was performed to remove the teratoma and the patient subsequently made a full recovery. Follow-up X-ray and computed tomography examinations were performed 6 months after surgery, which revealed no disease recurrence.
\end{abstract}

\section{Introduction}

Teratomas are a common tumor type that mainly occur in children and infants. The majority of cases are benignant, arising arise from the ovaries, retroperitoneum or sacrococcygeal region. However, a few cases are malignant and occur in middle age. Teratomas of the posterior mediastinum are uncommon tumors with a slow growth rate and a low incidence rate, accounting for only $8-13 \%$ of all tumors of the posterior mediastinum (1). They are mainly incidentally detected by chest imaging since there are no marked clinical symptoms. Treatment by surgical excision can achieve satisfactory results.

Situs inversus is a rare autosomal recessive disorder in which the organs are transposed from their normal location to the opposite side of the body (2). In India, the reported incidence of situs inversus is one case per 6,000-35,000 live births (3). The first known human case was reported by Fabricius in 1600 (2). The condition is termed situs inversus totalis when both the abdominal and thoracic viscera, including the dextrocardia, are involved. When present, it may be associated

Correspondence to: Dr Song Zhao, Department of Thoracic Surgery, The First Affiliated Hospital of Zhengzhou University, 1 Jianshe Road, Zhengzhou, Henan 450052, P.R. China

E-mail: songzhaocn@163.com

Key words: situs inversus totalis, teratoma, posterior mediastinum with several other abnormalities, including bronchiectasis, sinusitis and deficient tracheobronchial cilia, which is then known as Kartagener's syndrome. To the best of our knowledge, at present, the association between situs inversus totalis and teratoma has not been reported in the literature.

The present study describes the case of a 23-year-old woman who presented with situs inversus totalis and a mature teratoma in the posterior mediastinum.

\section{Case report}

In July 2014, a 23-year-old woman presented to The First Affiliated Hospital of Zhengzhou University with a 3-year history of intermittent chest tightness. A chest computed tomography (CT) scan and X-ray showed a mediastinal mass. The X-ray also revealed dextrocardia with a fundic gas shadow on the right side and a liver shadow on the left side (Fig. 1A and B). Contrast-enhanced CT was performed to obtain further information and revealed a posterior mediastinal complex cystic mass that was $10 \mathrm{~cm}$ in diameter (Fig. 1C). Following the radiological examination, a teratoma and situs inversus totalis were diagnosed. The physical examination showed that the patient was healthy with normal functional capacity since there was no fever, hypertension or abnormal respiration.

Surgery was performed to resect the teratoma. CT showed the presence of pleural effusion subsequent to the surgery (Fig. 1D). Grossly, the resected surgical specimen was a $9 \times 6.5 \times 3.5-\mathrm{cm}$ cystic mass, full of yellow pultaceous material and clear liquid (Fig. 2A and B). The wall of the cystic mass was observed to be divided by stratified squamous epithelium with an underlying simple ciliated columnar epithelium and sebaceous glands upon microscopy. Additionally, smooth muscle cells and adipose tissues were observed in the wall (Fig. 2C and D). A mature teratoma was diagnosed histologically since there was no evidence of malignancy. The patient exhibited a normal recovery after surgery and was discharged from hospital 9 days later. Follow-up was performed 6 months after surgery; X-ray examination and computed tomography scans revealed no residual mass or disease recurrence.

\section{Discussion}

Teratomas are a type of neoplasm arising from the pluripotent embryonic cells and containing three germ layers. No evident clinical symptoms of mediastinal teratomas exist 

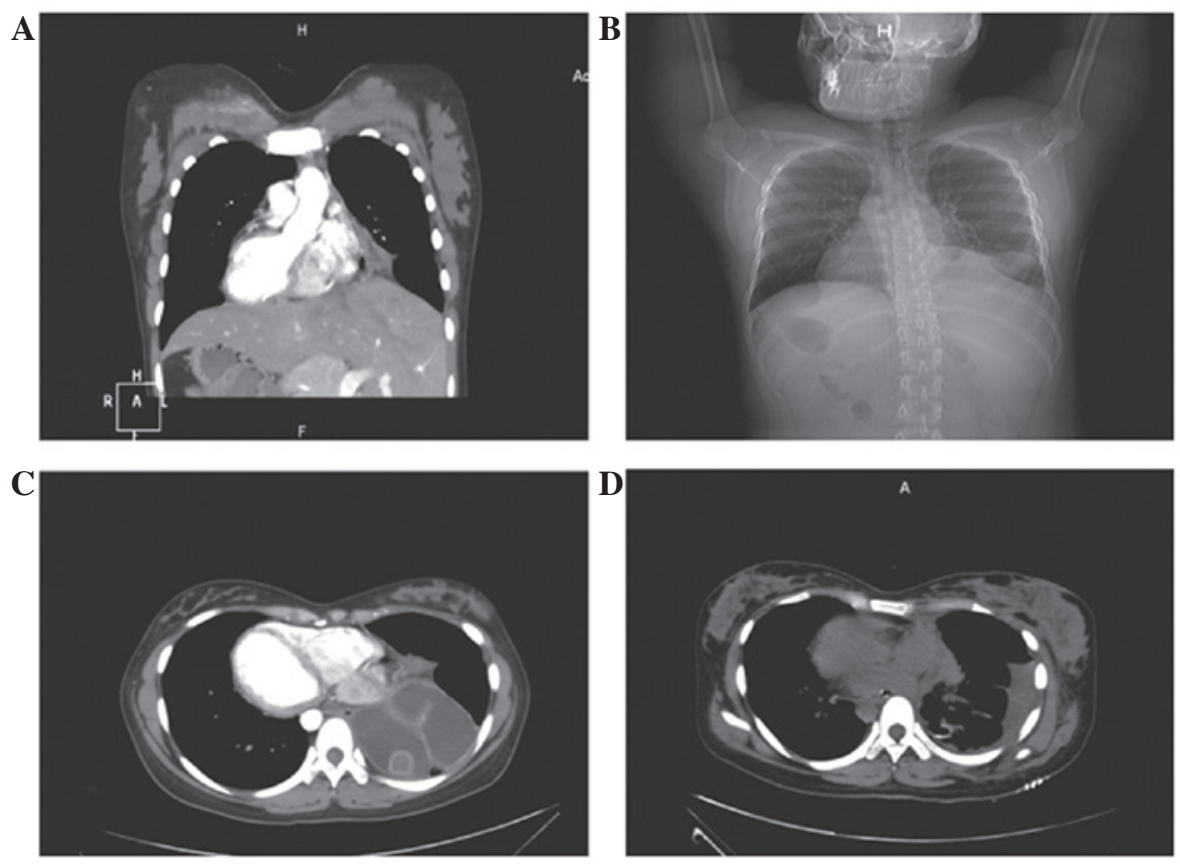

Figure 1. Computed tomography and X-ray images of a 23 -year-old female with a posterior mediastinal complex cystic mass and situs inversus totalis (A-C) prior to and (D) following surgery. Pleural effusion was present after surgery.
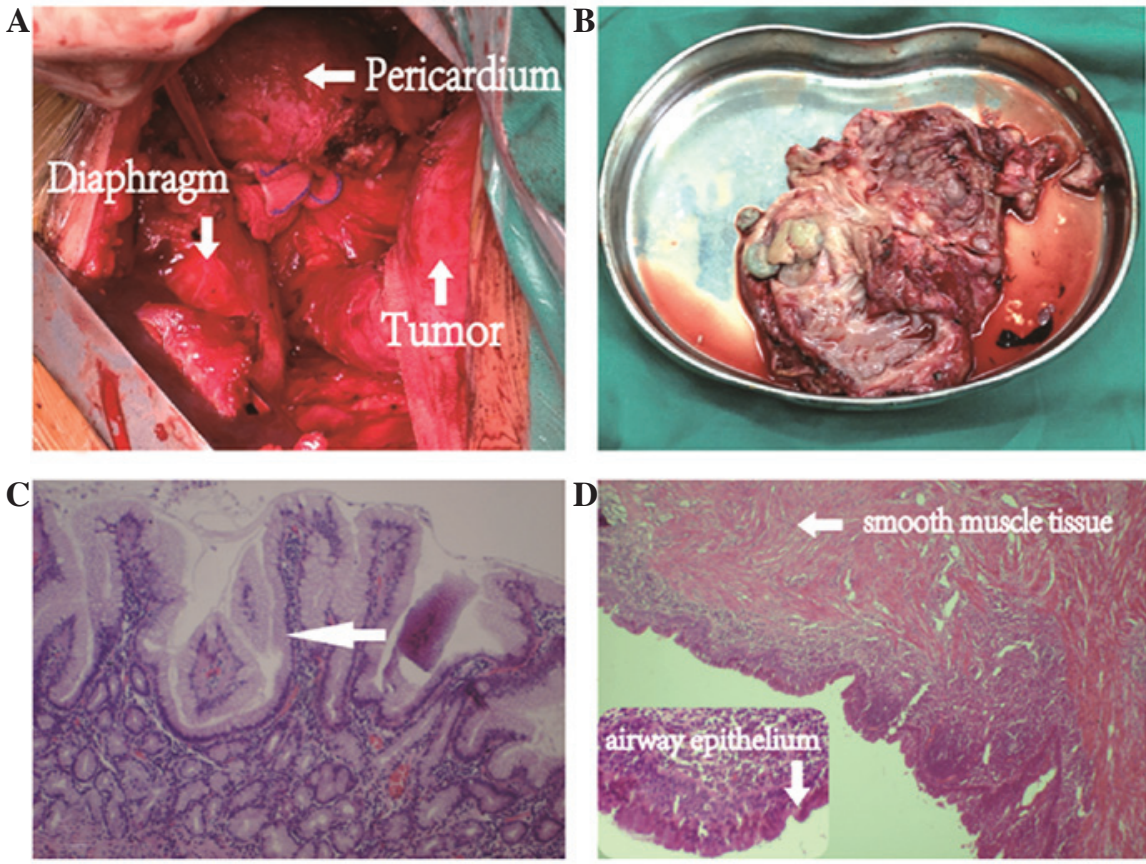

Figure 2. Gross and histological images of the tumor. (A) Location of the tumor during surgery. (B) Gross appearance of the tumor with a cystic region and granulated surface. Histopathological appearance of the tumor (hematoxylin and eosin staining; magnification, $\mathrm{x} 400$ ) containing $(\mathrm{C})$ intestinal epithelium, and (D) smooth muscle tissue and airway epithelium (inset).

until terminal stages. Teratomas in the mediastinum are often detected incidentally by chest X-ray. Pectoralgia, expiratory dyspnea and coughing are symptoms that may be caused by the compression of nearby structures. A rupture of the teratoma may cause superior vena cava syndrome or pneumonia, but occurs rarely (4). To the best of our knowledge, the current study represents the first case of a teratoma in the mediastinum in a patient with situs inversus totalis.
Surgical resection is the main choice for the treatment of a mediastinal teratoma since it aids in confirming the correct diagnosis and preventing complications. The majority of teratomas in the mediastinum are benign, while only $1-5 \%$ are malignant $(5,6)$. There are a number of indicators of malignancy in teratomas, such as invasion of the myocardium and great vessels (6). These indicators attest to the requirement for extensive surgery. The associated regions of the 
adherent mediastinal pleura and pericardium can be removed during the surgery. Since the majority of teratomas in the mediastinum are benign, a subtotal excision could achieve satisfactory results. With the progression of medical technology, optimal surgical results have been obtained (7).

Data on patients with situs inversus and peptic conditions, ulcer perforation, amoebic liver abscesses, acute cholecystitis, cholelithiasis, acute appendicitis or intestinal obstructions have been reported (8). However, with the exception of the present study, there have been no reports with regard to teratoma associated with situs inversus totalis. Establishment of a detailed and precise assessment prior to surgery is important in such cases.

In conclusion, this study is the first to report a patient with situs inversus totalis and posterior mediastinal teratoma. The patient was diagnosed via radiological methods and surgery was performed. The patient made a full recovery after surgical resection, and continues to attend follow-up examinations. We hypothesize that the occurrence of situs inversus totalis accompanied with posterior mediastinal mature teratoma in this patient may have been a coincidence, however, it may present a rare syndrome. To the best of our knowledge, no data regarding the association between situs inversus totalis and teratoma has been reported in the literature to date. Thus, similar cases must be reported in the future.

\section{References}

1. Anushree CN and Shanti V: Mature mediastinal teratoma. J Clin Diagn Res 9: ED05-ED06, 2015.

2. Pitiakoudis M, Tsaroucha AK, Katotomichelakis $M$, Polychronidis A and Simopoulos C: Laparoscopic cholecystectomy in a patient with situs inversus using ultrasonically activated coagulating scissors. Report of a case and review of the literature. Acta Chir Belg 105: 114-117, 2005.

3. Patel RB, Bhadreshwara K and Hukkeri S: Laparoscopic appendicectomy in a patient with situs inversus totalis. Indian J Surg 75 (Suppl 1): S41-S43, 2013.

4. Nichols CR: Mediastinal germ cell tumors. Clinical features and biologic correlates. Chest 99: 472-479, 1991.

5. Ousehal A, Skalli A, Nejjar M, Belaabidia B and Kadiri R: Malignant bilateral mediastinal teratoma: A case report. J Radiol 82: 174-176, 2001 (In French).

6. Levitt RG, Husband JE and Glazer HS: CT of primary germ-cell tumors of the mediastinum. AJR Am J Roentgenol 142: 73-78, 1984.

7. Tsubochi H, Endo S, Nakano T, Minegishi K, Tetsuka K and Hasegawa T: Extraction of mediastinal teratoma contents for complete thoracoscopic resection. Asian Cardiovasc Thorac Ann 23: 42-45, 2015.

8. Agarwal G and Kar DK: Teratoma of the anterior mediastinum presenting as a cystic neck mass: A case report. J Med Case Rep 2: 23, 2008 . 